\title{
Escleritis posterior bilateral
}

\section{Bilateral posterior scleritis}

\section{A. Zurutuza, J. Andonegui, L. Berástegui, N. Arruti}

\section{RESUMEN}

La escleritis posterior es un proceso inflamatorio de la parte posterior de la esclera. Su prevalencia es muy baja y el diagnóstico puede resultar complicado por la ausencia de signos oculares externos. Es más frecuente en mujeres. Cuando aparece en pacientes jóvenes no suele tener otras patologías asociadas, pero en mayores de 55 años hasta un tercio de los casos tienen relación con alguna enfermedad sistémica, sobre todo la artritis reumatoide. El diagnóstico de esta patología puede requerir un abordaje multidisciplinar y la colaboración de oftalmólogos con neurólogos, internistas o reumatólogos. En este artículo se describe un caso de escleritis posterior bilateral idiopática.

Palabras clave. Escleritis posterior. Idiopática. Bilateral.

\begin{abstract}
Posterior scleritis is an inflammatory process of the posterior part of the sclera. Its prevalence is very low and its diagnosis can be complicated due to the absence of external ocular signs. It is more frequent in women. In young patients it does not usually have other associated pathologies, but in those over 55 years nearly one-third of the cases have a relation with some systemic disease, above all rheumatoid arthritis. The diagnosis of this pathology can require a multidisciplinary approach and the collaboration of ophthalmologists with neurologists, internists or rheumatologists. This article describes a case of idiopathic bilateral posterior scleritis.
\end{abstract}

Key words. Posterior scleritis. Idiopathic. Bilateral.
Servicio de Oftalmología A.

Complejo Hospitalario de Navarra. Pamplona.

Recepción: 26 de marzo de 2011

Aceptación provisional: 11 de abril de 2011

Aceptación definitiva: 2 de mayo de 2011

\section{Correspondencia}

José Andonegui Navarro

Servicio de Oftalmología A

Complejo Hospitalario de Navarra

C/ Irunlarrea, 3

31008 Pamplona

E-mail: jandonen@cfnavarra.es 


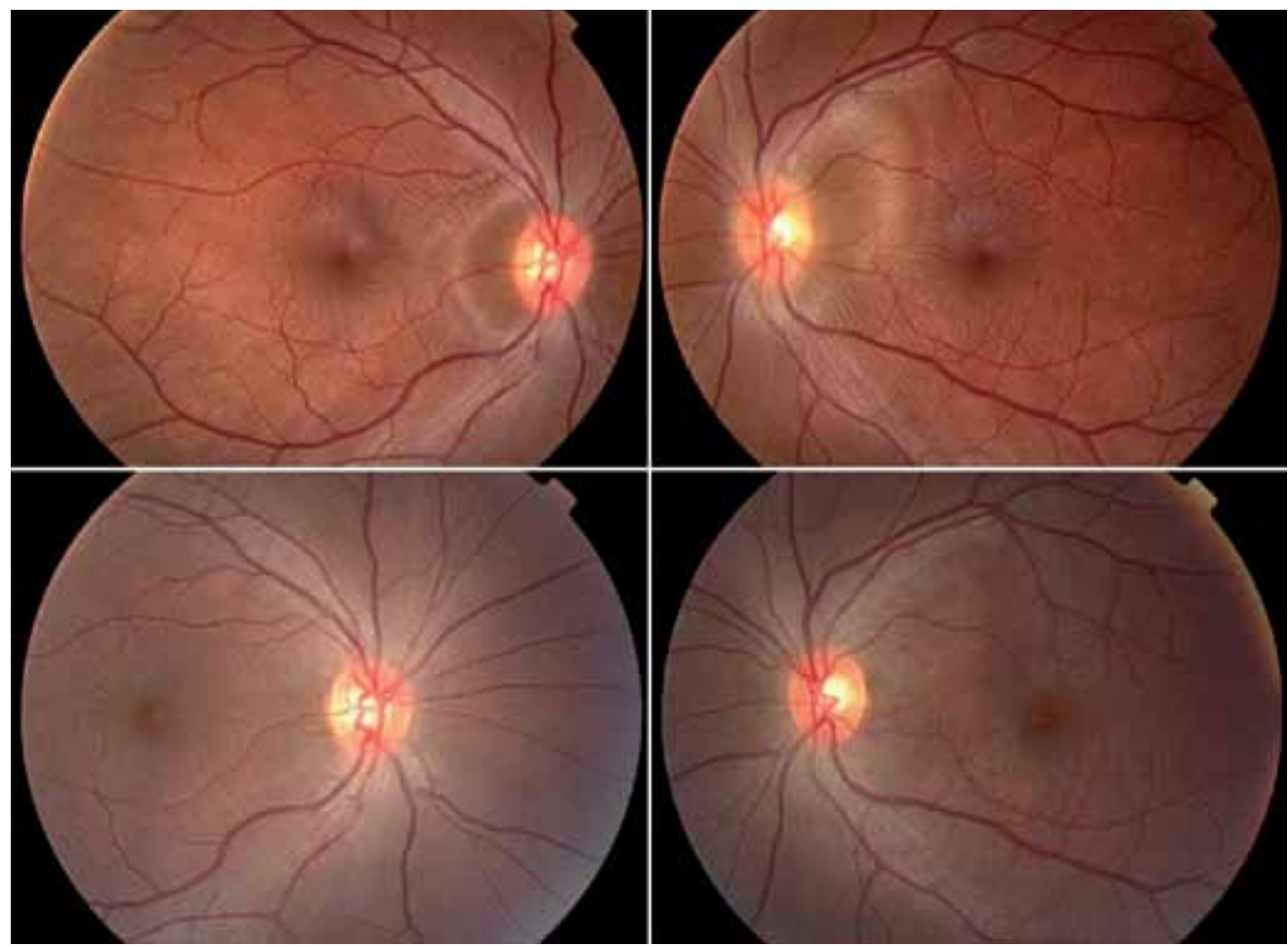

Figura 1. Arriba izquierda. Pliegues peripapilares y maculares en el fondo de ojo derecho. Arriba derecha. Pliegues peripapilares y maculares en el fondo de ojo izquierdo. Abajo izquierda. Desaparición de los pliegues después del tratamiento en ojo derecho. Abajo derecha. Desaparición de los pliegues después del tratamiento en ojo izquierdo.
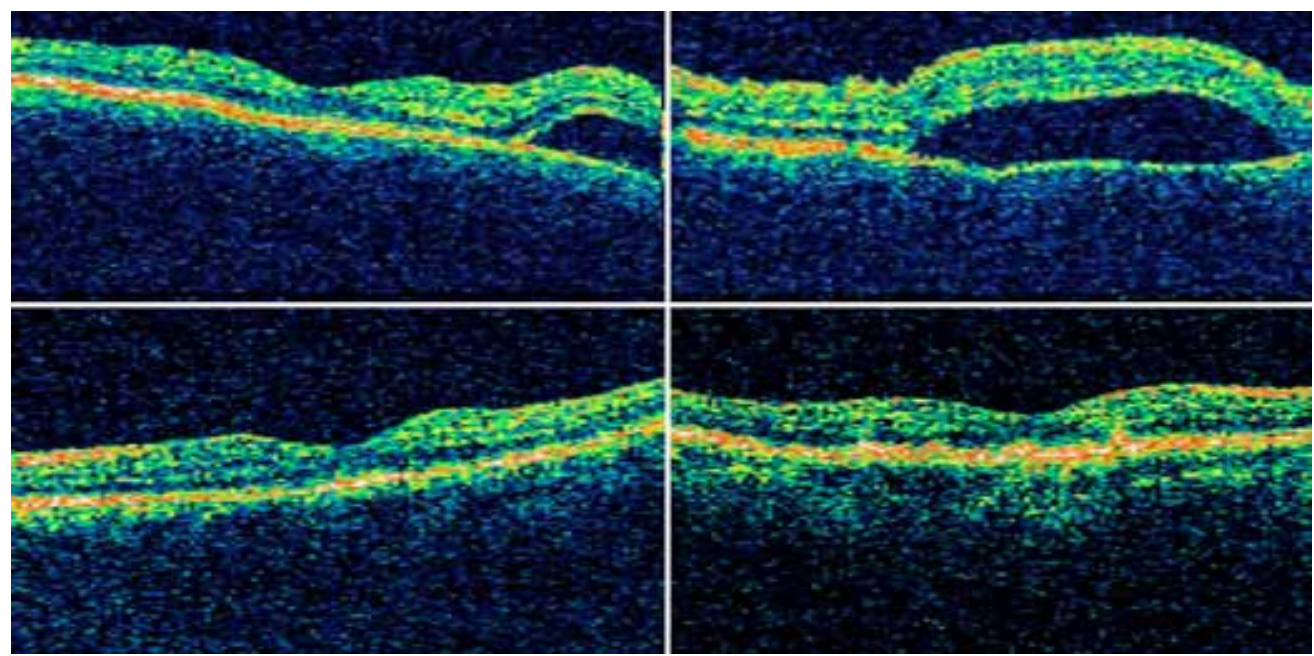

Figura 2. Arriba izquierda. Acúmulo de líquido subretiniano en la tomografía de coherencia óptica de ojo derecho. Arriba derecha. Acúmulo de líquido subretiniano en la tomografía de coherencia óptica de ojo izquierdo. Abajo izquierda. Desaparición del líquido subretiniano después del tratamiento en ojo derecho. Abajo derecha. Desaparición del líquido subretiniano después del tratamiento en ojo izquierdo. 


\section{INTRODUCCIÓN}

La escleritis posterior es una patología infrecuente que se presenta sobre todo en mujeres de edad media. Su diagnóstico puede resultar complejo puesto que a veces las manifestaciones oculares pueden ser poco aparentes o pueden verse enmascaradas por el dolor, uno de los síntomas más prominentes de esta entidad. La mayoría son idiopáticas, aunque $20-30 \%$ de los casos se asocian a alguna enfermedad sistémica siendo necesario en estos casos un abordaje multidisciplinar. En este artículo se describe un caso de escleritis posterior bilateral que fue tratado con éxito mediante corticoides vía oral.

\section{CASO CLÍNICO}

Mujer de 24 años con cefalea opresiva frontal y supraciliar de 11 días de evolución asociada a visión borrosa. En la exploración inicial realizada en urgencias se encontró una borrosidad papilar en su ojo izquierdo, por lo que fue remitida para ser valorada por los neurólogos. La exploración neurológica y el resto de la exploración física resultaron normales. La analítica sanguínea no mostró ninguna alteración relevante, siendo negativos el factor reumatoide y los
ANCA. También fue negativa la serología para Listeria, Bartonella, enfermedad de Lyme, Mycoplasma, virus de la Varicela, fiebre botonosa mediterránea, virus Herpes, VIH, Citomegalovirus, Toxoplasma, Epstein-Barr, brucelosis, sífilis y hepatitis. El TAC y la RNM, solicitados para descartar patología orbitaria o intracraneal, fueron normales, por lo que la paciente fue redirigida para ser evaluada por oftalmología.

En la exploración oftalmológica se encontró una agudeza visual de 0,8 en ojo derecho y 0,5 en ojo izquierdo. La presión intraocular era normal en ambos ojos. El ojo izquierdo presentaba un leve Tyndall, siendo el resto de la exploración del polo anterior normal en ambos ojos. En el fondo de ojo se observaron pliegues retinocoroideos en polo posterior con desprendimiento seroso peripapilar en ambos ojos. Asimismo se apreció una discreta congestión papilar con ligero borramiento de borde superior en su ojo izquierdo (Fig.1). La tomografía de coherencia óptica reveló un acúmulo de líquido subretiniano en el polo posterior en ambos ojos (Fig. 2). En la ecografía ocular modo B se apreció un engrosamiento escleral bilateral (Fig. 3). Con estos datos se estableció el diagnóstico de escleritis posterior bilateral idiopática y se instauró tratamiento con prednisona oral $60 \mathrm{mg} /$ día en pauta descendente, obteniendo en pocas semanas una buena respuesta clínica y anatómica tanto en el fondo de ojo (Fig. 1) como en la tomografía de coherencia óptica (Fig. 2).

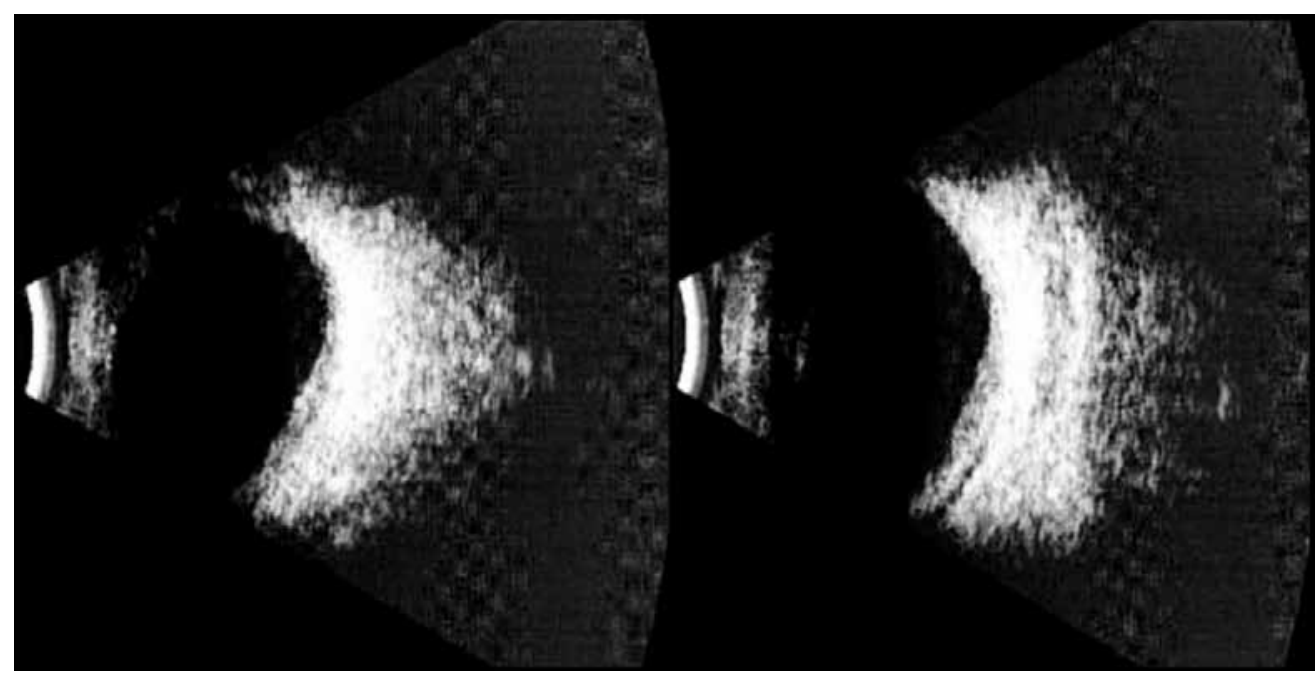

Figura 3. Izquierda. Engrosamiento escleral en la ecografía en modo B en ojo derecho. Derecha. Engrosamiento escleral en la ecografía en modo B en ojo izquierdo. 


\section{DISCUSIÓN}

La escleritis posterior es un proceso inflamatorio que afecta a la parte posterior de la esclera ${ }^{1}$. Su prevalencia en la población general es de 6 casos por 100.000 habitantes, aunque ha sido descrita en $0,2-6,3 \%$ de los pacientes con artirits reumatoide y hasta en un $7 \%$ de los pacientes con granulomatosis de Wegener ${ }^{2}$. Su baja prevalencia y la frecuente ausencia de signos oftalmoscópicamente detectables pueden dificultar su diagnóstico precoz.

La escleritis es una patología que afecta preferentemente a mujeres y se puede manifestar con un gran abanico de manifestaciones clínicas. Generalmente comienza antes de los 40 años. La mayoría son idiopáticas, aunque cuando aparece en mayores de 55, aproximadamente un tercio de los casos se asocian a alguna enfermedad sistémica. La asociación más frecuente es con la artritis reumatoide, aunque también puede aparecer en pacientes con lupus eritematoso sistémico, granulomatosis de Wegener o poliarteritis nodosa $a^{3}$. La escleritis puede ser la primera y/o única manifestación inicial de estas enfermedades sistémicas, por lo que el diagnóstico debe ser lo más correcto y precoz posible para la instauración del tratamiento sistémico ya que éste puede detener la progresión del proceso tanto ocular como sistémico.

La inflamación y engrosamiento de la esclera posterior provoca un aplanamiento posterior del globo ocular que es detectable en el fondo de ojo por la aparición de pliegues coroideos. No es raro visualizar un desprendimiento neurosensorial y un edema de papila asociado ${ }^{4}$, como es el caso de nuestra paciente. El dolor es el síntoma más común en la escleritis posterior y es el resultado de la compresión y estimulación directa de las terminaciones nerviosas provocadas por la inflamación, por lo que es considerado como el mejor indicador de la actividad inflamatoria. En ocasiones el dolor puede ser severo y aumentar a la palpación, irradiándose a otras zonas de la cabeza y pudiendo despertar por la noche al paciente, si bien es cierto que se han re- gistrado casos indoloros ${ }^{5}$. La disminución de la agudeza visual está justificada por los signos detectables a nivel del polo posterior y papila, aunque también puede deberse a la hipermetropización provocada por el engrosamiento escleral y el acortamiento de la longitud axial del globo ocular.

La ecografía en manos experimentadas es la prueba principal para el diagnóstico de la escleritis posterior, observando en ella un aumento del grosor escleral y el clásico signo de la $\mathrm{T}$, que representa la inflamación escleral y un acúmulo de líquido en el espacio subtenoniano adyacente ${ }^{6}$. También la resonancia magnética y el escáner pueden ser útiles ${ }^{7}$.

El tratamiento de la escleritis posterior se basa en corticoides orales en dosis de $1 \mathrm{mg} / \mathrm{kg} /$ día en una sola toma matutina ${ }^{8}$. También los AINE pueden ser útiles, pero en general en la escleritis posterior se recomienda utilizar de entrada los corticoides orales. Si la enfermedad se presenta como especialmente agresiva se puede tratar mediante pulsos de metilprednisolona intravenosa, a dosis de $1 \mathrm{~g} /$ día durante tres días, seguido de corticoides orales a la dosis ya indicada ${ }^{2}$. Si no se observa respuesta terapéutica a los corticoides se recomienda el uso de inmunosupresores sistémicos como un antimetabolito (metotrexate) ${ }^{9}$, un inmunomodulador (ciclosporina) ${ }^{10} \mathrm{o}$ un fármaco citotóxico (ciclofosfamida), vigilando estrechamente la aparición de posibles efectos secundarios.

Si no se tiene un alto índice de sospecha el diagnóstico de escleritis posterior puede resultar difícil. En nuestra paciente la ausencia de signos oculares externos y el dolor, unidos a la borrosidad papilar en su ojo izquierdo, hicieron sospechar inicialmente en un proceso neurológico. Un examen ocular más detallado después de que la evaluación neurológica resultase normal orientó el diagnóstico hacia esta entidad. Habiéndose descartado mediante la analítica y la exploración otras patologías asociadas se concluyó que se trataba de una escleritis posterior bilateral idiopática. No solo los oftalmólogos, también los neurólogos, reumatólogos o internistas de- 
ben tener en mente la posibilidad de esta enfermedad, sobre todo en pacientes con algunas de sus patologías asociadas. La colaboración y el examen multidisciplinar resultan esenciales para el adecuado manejo de este proceso.

\section{BIBLIOGRAFÍA}

1. Dubord PJ, Chalmers A. Scleritis and epiescleritis: diagnosis and management. Focal Points: Clinical Modules for Ophthalmologists. San Francisco: American Academy of Ophthalmology; 1995, module 9.

2. GALOR A, THORNe JE. Scleritis and peripheral ulcerative queratitis. Rheum Dis Clin N Am 2007; 33: 835-854.

3. KANSKI JJ. Episclera and sclera. En: Kanski JJ, editor. Clinical Ophthalmology. A systematic approach. Sixth edition. Edinburg: Butterworth Heinemann Elsevier 2007; 323-335.

4. GRIEBEL SR, KosmorsKy GS. Choroidal folds associated with increased intracranial pressure. Am J Ophthalmol 2001; 131: 158-159.
5. SaiKia P, Nashed A, Helbig H, Hillenkamp J. Bilateral posterior escleritis: an idiopathic painless presentation. Ocul Immunol Inflamm 2010; 18:452-453.

6. Machado DO, Curi AL, Bessa TF, Campos WR, ORÉFICE F. Posterior scleritis: clinical features, systemic association, treatment and evolution of 23 patients. Arq Bras Oftalmol 2009; 72: 321-326.

7. Chanques VJ, Lam S, Tessler HH, Mafee MG. Computed tomography and magnetic resonance imaging in the diagnosis of posterior scleritis. Ann Ophthalmol 1993; 25: 84-94.

8. Smith JR, Mackensen F, Rosenbaum JT. Therapy insight: scleritis and its relationship to systemic autoimmune disease. Nat Clin Pract Rheumatol 2007; 3: 219-226.

9. Gangaputra S, Newcomb CW, Lieseqanq TL, Kacmaz RO, JABS DA, LEVY-CLARKE GA et al. Methotrexate for ocular inflammatory diseases. Ophthalmology 2009; 116: 2188-2198.

10. Kacmaz RO, Kempen JH, Newcomb C, Daniel E, Gangaputra S, Nussenblatt RB et al. Cyclosporine for ocular inflammatory diseases. Ophthalmology 2010; 117: 576-584. 
